\section{RpoS proteolysis is controlled directly by ATP levels in Escherichia coli}

\author{
Celeste N. Peterson, ${ }^{1,2,3}$ Igor Levchenko, ${ }^{2,4}$ \\ Joshua D. Rabinowitz, ${ }^{1}$ Tania A. Baker, ${ }^{2,4}$ \\ and Thomas J. Silhavy ${ }^{1,5}$
}

\begin{abstract}
${ }^{1}$ Department of Molecular Biology, Princeton University, Princeton, New Jersey 08544, USA; ${ }^{2}$ Department of Biology, Massachusetts Institute of Technology, Cambridge, Massachusetts 02139, USA; ${ }^{3}$ Department of Biology, Suffolk University, Boston, Massachusetts 02108, USA; ${ }^{4}$ Howard Hughes Medical Institute, Massachusetts Institute of Technology, Cambridge, Massachusetts 02139, USA
\end{abstract}

The master regulator of stationary phase in Escherichia coli, RpoS, responds to carbon availability through changes in stability, but the individual steps in the pathway are unknown. Here we systematically block key steps of glycolysis and the citric acid cycle and monitor the effect on RpoS degradation in vivo. Nutrient upshifts trigger RpoS degradation independently of protein synthesis by activating metabolic pathways that generate small energy molecules. Using metabolic mutants and inhibitors, we show that ATP, but not GTP or NADH, is necessary for RpoS degradation. In vitro reconstitution assays directly demonstrate that ClpXP fails to degrade RpoS, but not other proteins, at low ATP hydrolysis rates. These data suggest that cellular ATP levels directly control RpoS stability.

Supplemental material is available for this article.

Received November 17, 2011; revised version accepted February 9, 2012.

RpoS, also known as $\sigma^{\mathrm{S}}$ or $\sigma^{38}$, is an alternate $\sigma$ factor that serves as the master regulator of stationary phase and the general stress response in many proteobacteria (Loewen and Hengge-Aronis 1994; Dong and Schellhorn 2009). In Escherichia coli, it has been reported to control transcription of up to $10 \%$ of the genome (Weber et al. 2005) and is necessary for survival in numerous environments (McCann et al. 1991). In healthy growing cells, RpoS is still produced but is immediately directed by the adaptor protein SprE (RssB) to the AAA ${ }^{+}$ATPase ClpXP protease for unfolding and degradation (Muffler et al. 1996; Pratt and Silhavy 1996; Baker and Sauer 2012). When carbon sources are depleted, RpoS proteolysis ceases, causing it to rapidly accumulate and compete with other $\sigma$ factors for binding to the core RNA polymerase. This $\sigma$ exchange in turn leads to changes in gene expression that protect against stresses

[Keywords: protein unfolding; metabolic state; cellular energy; stationary phase]

${ }^{5}$ Corresponding author.

E-mail tsilhavy@princeton.edu.

Article is online at http://www.genesdev.org/cgi/doi/10.1101/gad.183517.111. and prepare the cell for long-term survival. Conversely, when carbon sources are replenished, RpoS is actively degraded, and RpoS-controlled genes are no longer expressed. We wish to understand the molecular signals that control RpoS stability.

There are several plausible mechanisms of RpoS regulation. SprE is an adaptor specific for RpoS degradation and belongs to the response regulator family of twocomponent signaling proteins. It has a highly conserved $\mathrm{N}$-terminal receiver domain with a conserved aspartic acid, D58, so it was possible that its activity was controlled by phosphorylation of this site. However, it is an orphan response regulator; there is no cognate sensor kinase. We and others have mutated the phosphorylation site of the chromosomal allele of $\operatorname{sprE}$ and found that RpoS proteolysis was still regulated upon carbon starvation in a SprE-dependent manner (Peterson et al. 2004; Zhou and Gottesman 2006). Accordingly, we conclude that phosphorylation of SprE is not integral to the carbon starvation signaling pathway that regulates RpoS. SprE was recently found to regulate mRNA stability (Carabetta et al. 2009), and it is not known if phosphorylation plays a role in that activity.

In response to other stresses, such as phosphate starvation, magnesium starvation, and DNA damage, a major mechanism for RpoS accumulation is antagonism of the adaptor SprE by the anti-adaptor proteins IraP, IraM, and IraD, respectively (Bougdour et al. 2006, 2008; Merrikh et al. 2009). However, none of the Ira proteins have been associated with carbon starvation, and despite extensive screens, no other proteins have been identified in the carbon starvation signaling pathway. Although the Clp protease is active during starvation and its levels remain constant (Damerau and St John 1993; Schweder et al. 1996), the ability of the protease to degrade RpoS can be antagonized by the presence of other substrates. Mutants affecting the production of mistranslated proteins showed that RpoS accumulation is partially due to Clp sequestration by aberrant oxidized proteins made during carbon starvation (Fredriksson et al. 2007). Although nitrogen starvation and carbon starvation both result in the production of similar amounts of oxidized proteins (Ballesteros et al. 2001), RpoS accumulates to a dramatically higher level during carbon starvation (Mandel and Silhavy 2005). This observation suggests that an additional mechanism must exist that stabilizes RpoS during carbon starvation that has yet to be identified.

Previously, we showed that SprE-dependent degradation of RpoS begins very quickly after the addition of glucose to carbon-starved cells (Peterson et al. 2004). As opposed to nutrient depletions, nutrient upshifts provide a convenient experimental method for investigating the degradation reaction in vivo. Using this method, we show here that RpoS degradation requires active metabolismin particular, the generation of ATP. Experiments with purified components in vitro confirm RpoS degradation is more sensitive to ATP levels than are other substrates. We suggest that the ability to sense cellular energy levels is a property that is built into the three partners-RpoS, SprE, and ClpXP-working together in an ATP-dependent manner to control RpoS levels during the entry and exit from starvation. 


\section{Results and Discussion}

\section{RpoS degradation requires a carbon source} that can be metabolized

To measure RpoS stability, new synthesis was prevented by the addition of chloramphenicol, and levels of RpoS were monitored by Western blot over time. In starved cells with no de novo protein synthesis, RpoS remained stable for hours. Thus, the synthesis of aberrant proteins (or any new proteins) was not required to maintain RpoS stability in starved cells. However, RpoS degradation commenced immediately upon the addition of glucose, and complete destruction took only a few minutes (Fig. 1). The speed at which this degradation initiated raised the possibility that a small molecule or mechanical signal may be the cellular indicator of carbon source availability.

Glucose and phosphate transport into the cell is monitored by the proteins GlcIIa and PhoU, respectively (Deutscher et al. 2006; Rice et al. 2009), and the slowing of phosphate transport upon phosphate limitation signals production of a small RNA that stimulates rpoS translation (Ruiz and Silhavy 2003). To test whether metabolite transport alone could trigger RpoS degradation, the glucose analog $\alpha$-methylglucoside was added to starved cells. This analog is transported across the cytoplasmic membrane by the phosphotransfer system (PTS) like glucose, but once inside the cell, it cannot be metabolized. Upon addition of $\alpha$-methylglucoside, RpoS degradation was not stimulated, whereas upon addition of glucose, RpoS was rapidly degraded (Supplemental Fig. 1). Our results are in agreement with previous studies reporting that mutations in the PTS affect RpoS transcription and translation but not stability (Cunning and Elliott 1999). Thus, we conclude that carbon substrates must be actively metabolized to initiate RpoS degradation.

\section{The generation of ATP is required for RpoS degradation}

The observation that RpoS degradation requires glucose metabolism (Supplemental Fig. 1) suggested that some central metabolite signals carbon source availability. To identify such a molecule, we used a combination of alternative carbon sources and mutations that block metabolic pathways at key places.

We first determined whether pyruvate or TCA metabolites were sufficient for signaling by using mutations

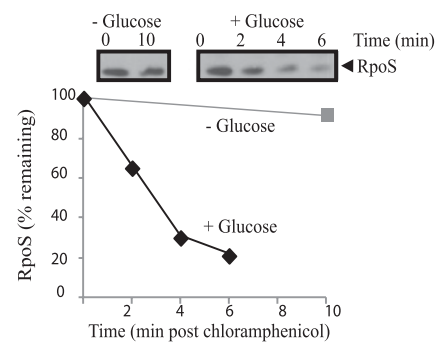

Figure 1. Activation of RpoS degradation is rapid and proceeds without de novo protein synthesis. Exponentially growing cells in M63 minimal medium were subject to immediate starvation for $1 \mathrm{~h}$. Translation was inhibited by addition of chloramphenicol for $1 \mathrm{~min}$, and then either no glucose was added or glucose was added to a final concentration of $0.4 \%$. Minutes indicate the time passed after addition of chloramphenicol. All samples were taken for Western blot preparation as described in the Materials and Methods. A representative gel from duplicate experiments is shown. that impeded gluconeogenesis. Mutations in ppsA and $p c k A$ block the synthesis of phosphoenolpyruvate from pyruvate and oxaloacetate, respectively (Supplemental Fig. 2A). In minimal medium, this double mutant strain grows well if glucose is provided. However, it cannot grow in minimal medium with pyruvate because it cannot produce the glucose necessary for essential biosynthetic processes such as peptidoglycan and nucleotide synthesis (Supplemental Fig. 2A). Using this strain, we determined whether pyruvate and the citric acid (TCA) cycle metabolites were sufficient to trigger RpoS degradation. Cells were grown in minimal glucose with $0.005 \%$ pyruvate added to induce the pyruvate transport systems and starved for $1 \mathrm{~h}$, after which $0.4 \%$ pyruvate was added. RpoS turnover commenced upon pyruvate addition in both the wild-type background $146 \%$ of the protein remained after $10 \mathrm{~min}$ ) and the double mutant blocked for gluconeogenesis $(16 \%$ of the protein remained after 10 min), although degradation was not as fast for the wildtype strains as observed in glucose (Supplemental Fig. 2A). Thus, pyruvate or metabolites derived from it in the TCA cycle can trigger RpoS degradation.

The experiments described above establish that pyruvate metabolism is sufficient for RpoS degradation, but do not prove whether it is necessary. To test whether glycolytic metabolites were also sufficient to activate RpoS degradation, we used a mutant in the glt $A$ gene that encodes for citrate synthase, the first enzyme in the citric acid cycle. This strain is an auxotroph for glutamate and cannot synthesize any of the metabolites in the right half of the TCA cycle, but can carry out glycolysis and the left side of the TCA cycle (Supplemental Fig. 2B). When glucose was added to a starved gltA::kan strain, RpoS degradation commenced immediately, as it does in wildtype. Ten minutes after adding glucose, 9\% of RpoS remained in the wild-type strain, while $5 \%$ remained in the gltA mutant strain (Supplemental Fig. 2B). Thus, glycolytic metabolites and the left half of the TCA cycle suffice to activate RpoS proteolysis. To determine whether pyruvate was sufficient, we used the same gltA::kan mutant (Supplemental Fig. 2C). When pyruvate was added to this starved gltA::kan strain, RpoS degradation was impaired. Ten minutes after adding pyruvate, $52 \%$ of RpoS remained in the wild-type strain, but $99 \%$ remained in the gltA mutant strain (Supplemental Fig. 2C). Therefore, pyruvate and acetyl-CoA per se were not sufficient to activate RpoS degradation.

Taken together, the glt $A$ mutant results suggest that glycolytic activity can produce the degradation signal, as can pyruvate oxidation via the TCA cycle. In contrast, addition of pyruvate without the ability to oxidize it via the right side of the TCA cycle is insufficient. Thus, the signal is not one particular central metabolite, but something that is produced by either glycolysis (Supplemental Fig. 2B,C) or the full activity of the TCA cycle (Supplemental Fig. 2A). Candidate metabolites produced by both processes are the central energy-transducing molecules.

There are two types of energy-transducing molecules derived from glycolysis and the citric acid cycle: ATP and NADH. Both have been shown to increase markedly during nutrient upshifts. Both molecules may also be converted into other energy-transducing molecules, such as GTP or NADPH, but as a first step, we sought to identify which general class is responsible for activating RpoS degradation. 
One way to distinguish between the contributions of ATP and NADH was to use CCCP (carbonyl cyanide $m$-chlorophenylhydrazone), which blocks oxidative phosphorylation by disrupting the proton motive force. In the presence of this uncoupler, NADH and other reduced molecules generated by the TCA cycle cannot be converted to ATP. Therefore, in the presence of CCCP, succinate added to starved cells increases NADH levels but not ATP levels. In contrast, glucose generates not only NADH, but also ATP, due to substrate-level phosphorylations in glycolysis (Fig. 2A; Zhang and Haldenwang 2005).

CCCP addition had no effect on RpoS stability in starved cells (data not shown). In the absence of CCCP, succinate stimulated RpoS proteolysis. Strikingly, however, when CCCP was present to inhibit the conversion of NADH to ATP, succinate addition did not activate RpoS

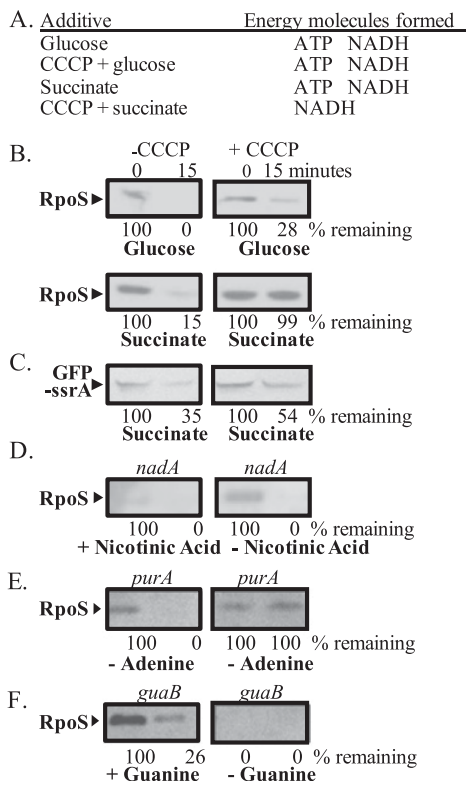

Figure 2. ATP is required to trigger RpoS degradation. $(A)$ Summary of the energy molecules generated when either glucose or succinate is added to starved cells in the presence or absence of CCCP (an uncoupler that disrupts the proton motive force). $(B)$ RpoS proteolysis is initiated when $0.4 \%$ glucose is added to starved cells, even in the presence of CCCP. Succinate $(0.4 \%)$ can trigger RpoS degradation but not in presence of CCCP, when the NADH cannot be converted to ATP. Numbers below the Western blot are a quantification of the band intensity, normalized to $100 \%$ for the time $=$ 0 samples. A representative gel from duplicate experiments is shown. Minutes refers to time passed after addition of carbon sources. $(C)$ Degradation of another ClpXP substrate in low ATP conditions caused by the CCCP and succinate. Substrate GFP-ssrA degradation as monitored by Western blot after $0.4 \%$ succinate addition with and without CCCP. $(D-F)$ Westerns are not from starvation experiments, but rather depletion experiments of essential components involved in either ATP or NADH biosynthesis. Cells were grown until mid-log stage in M63 supplemented with an essential component. They were washed twice, starved for the specific component, and treated with chloramphenicol. RpoS degradation was followed by Western blot. (D) RpoS degradation following a 2- $\mathrm{h}$ depletion of nicotinic acid, the precursor of NADH in nadA mutant strains. $(E)$ RpoS degradation following a 1- $\mathrm{h}$ depletion of adenine, the precursor of ATP in purA mutant strains. $(F)$ RpoS degradation following a 1-h depletion of guanine, the precursor to GTP in guaB mutant strains. Depletion of guanine in the guaB mutant strain leads to a sharp increase in ATP levels. degradation (Fig. 2B). Thus, NADH accumulation alone was not sufficient to trigger RpoS degradation. In contrast, when glucose was added to cells treated with the uncoupler, RpoS degradation commenced, although at a slightly lower rate than without CCCP (Fig. 2B), likely reflecting the lowered production of ATP under these conditions. These data demonstrated that changes in the proton motive force itself are not responsible for triggering RpoS degradation. Importantly, addition of the uncoupler to cells metabolizing succinate did not inactivate ClpXP. Degradation of the control protein GFP-ssrA still occurred under these conditions, although at a slower rate than in the presence of succinate alone (Fig. 2C). Thus, RpoS degradation was more sensitive to ATP levels than degradation of other well-characterized ClpXP substrates.

We further confirmed that ATP levels but not NADH levels affected RpoS stability by using mutant strains defective in biosynthesis of these specific metabolites. A nadA mutant that is defective for an enzyme necessary for NAD production was grown in medium supplemented with nicotinic acid to bypass the biosynthetic block. When cells were in exponential phase, they were depleted of NAD by resuspension in minimal glucose medium without nicotinic acid, and the RpoS half-life was monitored. After $2 \mathrm{~h}$ of NAD depletion, RpoS remained unstable, despite growth being impaired (Fig. 2D). The levels of RpoS at the time 0 samples were slightly higher in the absence of NAD than in its presence. This effect could be due to the increase in RpoS synthesis that follows the slower growth rate during the depletion; nevertheless, RpoS turnover was rapid in these conditions, so the effect was independent of the carbon starvation pathway.

In contrast, when cells were depleted of a precursor to ATP, RpoS was stabilized. A strain carrying a mutation in the purA gene cannot synthesize adenine, the ATP precursor, and can only grow when supplemented with the metabolite. When cells were resuspended in minimal glucose without adenine, there was little degradation of RpoS after $1 \mathrm{~h}$ of depletion (Fig. 2E). We also used a guaB mutant strain that is deficient in the synthesis of GTP. When these cells were grown in the presence of guanine, RpoS was still somewhat unstable. In the absence of guanine, the guaB strain has increased levels of ATP (Schneider and Gourse 2004), and after $1 \mathrm{~h}$ of depletion, RpoS levels were too low to be detected in our experiments (Fig. 2F).

Unlike the experiments where we added a carbon source, in these experiments, we removed an essential nutrient. The nicotinic acid and adenine depletion experiments are therefore representative of a downshift, while the upshift experiments described earlier measured the exit from starvation transition. Although there may be minor differences between the two pathways, we conclude that both pathways use ATP or a similar molecule derived from ATP to control RpoS proteolysis.

ATP is a compelling candidate for the starvation signal for several reasons. First, ATP is the energy currency of the cell. It is a known signal for starvation for stationary phase in other organisms, such as Bacillus subtilis (Zhang and Haldenwang 2005). Furthermore, ATP levels are highly correlated with RpoS degradation: ATP levels increase fivefold in the $5 \mathrm{~min}$ after carbon replenishment, when RpoS is being degraded (Murray et al. 2003). ATP levels are 
also transiently reduced and RpoS is transiently stabilized during late exponential phase during the diauxic shift (Fischer et al. 1998). Finally, ATP levels also drop during phosphate starvation (Kwon et al. 2010). This drop likely accounts for the RpoS stabilization during phosphate starvation that is independent of the anti-adaptor IraP (Bougdour et al. 2006).

\section{In vitro RpoS degradation requires high ATP levels}

RpoS degradation has been reconstituted in vitro from the purified components RpoS, SprE, ClpXP, and ATP (Klauck et al. 2001; Zhou et al. 2001). Hence, we already know that ATP is important for degradation. In accordance with the genetic and physiological studies above, our model predicts that RpoS degradation in vitro would be unusually sensitive to ATP levels.

Most ClpXP substrates are degraded across a wide range of ATP concentrations, although data from concentrations $<50 \mu \mathrm{M}$ are unreliable due to destabilization of the ClpX hexamer. Translocation by ClpXP is typically processive, so that once the protease has begun to unfold a molecule, it continues to unfold and completely degrade it, even at low ATP concentrations. The protein TitinV15PssrA is a model ClpXP substrate that has been extensively characterized. It is a tightly wound protein that requires numerous rounds of ATP hydrolysis before degradation. Despite this feature, ClpXP proteolysis of TitinV15P-ssrA perpetuates at low ATP concentrations (Fig. 3A). Even at $50 \mu \mathrm{M}$ ATP, TitinV15P-ssrA degradation was robust and no detectable substrate remained after $16 \mathrm{~h}$. However, a small subset of ClpXP substrates, including the MuA transposase tetrameter and some variants of GFP-ssrA, is not degraded in low ATP conditions (Martin et al. 2008; Nager et al. 2011; AH Abdelhakim and TA Baker, unpubl.). RpoS fell into this category of substrates, as degradation failed at $50 \mu \mathrm{M}$ ATP, and after $16 \mathrm{~h}$, nearly $90 \%$ of the

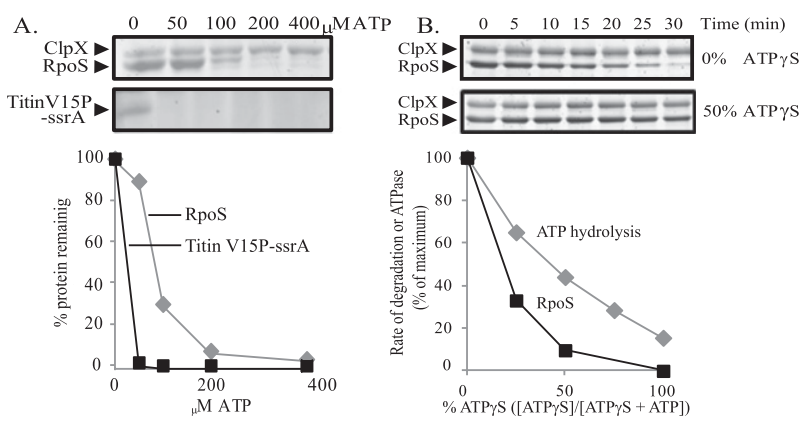

Figure 3. In vitro RpoS degradation only proceeds robustly under high ATP hydrolysis rates. (A) At low ATP levels, ClpXP degradation of the model substrate TitinV15P-ssrA proceeds, but degradation of RpoS fails. RpoS $(5 \mu \mathrm{M})$ was incubated overnight with $\mathrm{ClpX}_{6}(0.3$ $\mu \mathrm{M})$, $\mathrm{ClpP}_{14}(0.9 \mu \mathrm{M})$, phosphorylated $\operatorname{SprE}(0.1 \mu \mathrm{M})$, an ATP regeneration mix, and different concentrations of ATP before assaying degradation by SDS page and quantification. A representative gel from duplicate experiments is shown. ClpX runs much slower than TitinV15P-ssrA and is in the portion of the gel that is not included in the figure. (B) Increasing ATP $\gamma$ S concentration slows the ATP hydrolysis rates in an approximately linear fashion but disrupts RpoS degradation rates in a nonlinear manner. ATPase rates were monitored by following the reduction of $\mathrm{NADH}$ in an enzyme couple reaction. Degradation reactions were carried out with the same reaction conditions as in $A$, except that ATP and ATP $\gamma$ S combinations were used and the ATP regeneration mix was not present. protein remained (Fig. 3A). Degradation of RpoS was also impaired at $100 \mu \mathrm{M}$ and even $200 \mu \mathrm{M}$ ATP. Although we did not directly measure ATP concentrations in our in vivo experiments, they have been measured multiple times in the past under similar culture conditions. The in vitro ATP concentrations at which RpoS degradation failed are on the same order of magnitude as cellular levels measured in vivo during starvation, although comparing $K_{M}$ values in vitro and in vivo can be complicated (Burton et al. 2003; Schneider and Gourse 2004; Gigliobianco et al. 2010). In summary, in vitro RpoS degradation was clearly more sensitive to ATP concentrations than some or most other ClpXP substrates.

At low concentrations of ATP, ClpX hydrolyzes ATP more slowly. In addition to the endpoint experiments, we used kinetic measurements to confirm that the rate of RpoS degradation was dependent on robust ATP hydrolysis. The ATP analog ATP $\gamma$ S slows down ClpX ATPase activity but has not been shown to affect the binding of substrates to the protease (Hersch et al. 2005). Decreasing the ATP:ATP $\gamma$ S ratio had an approximately linear effect. For RpoS, there was a nonlinear effect of increasing ATP $\gamma S$ on the degradation rate; a small decrease in the ATPase rate caused by the addition of ATP $\gamma S$ resulted in a dramatic decrease in RpoS proteolysis such that an equal mixture of the two forms caused $>90 \%$ reduction in degradation rates (Fig. 3B). This nonlinear effect is similar to what has been observed with other stalling substrates (Martin et al. 2008; Nager et al. 2011). Thus, RpoS belonged to the category of proteins whose degradation is critically dependent on ATP hydrolysis rates.

\section{Conclusion}

In response to carbon availability, the master regulator of stationary phase, RpoS, is controlled by proteolysis. However, the molecular mechanisms connecting carbon levels to degradation had not been identified. Here we combined in vivo experiments with mutations and inhibitors that block key steps of central metabolism and demonstrate that activating RpoS proteolysis upon carbon upshifts does not require de novo gene expression but that it is more dependent on small energy molecules such as ATP than other ClpXP substrates. Further tests using anabolic mutants showed that RpoS proteolysis is sensitive to ATP but not GTP or NADH levels. Finally, reconstitution experiments in vitro demonstrated that RpoS degradation failed at low ATP hydrolysis rates, while degradation of other substrates continued. Collectively, these data suggest that RpoS proteolysis is directly controlled by ATP levels and that upon carbon upshifts, cellular ATP concentrations rise (Murray et al. 2003; Schneider and Gourse 2004) and empower ClpXP-mediated RpoS degradation. By bypassing slow gene expression cascades that involve long-lived components in low numbers and instead connecting RpoS proteolysis directly to ATP levels, cells ensure the most reliable and fastest response to changes in nutrient conditions.

The exact biochemical mechanism by which RpoS degradation depends on ATP levels still remains to be worked out in detail, but we suggest that it is a reflection of how all three partners-the substrate, adaptor, and protease-stall proteolysis at low ATP levels. In vivo observations suggest that the Clp/RpoS/SprE ternary complex is still formed during stationary phase (Becker et al. 2000) but does not proceed to degradation. Detailed 
biochemical studies of the workings of the ClpXP protease have in turn shown that substrate engagement in degradation critically depends on the active function of ClpXP. Reducing ATP levels slows ClpX translocation, making it more likely that partially unfolded intermediates accumulate in sufficient quantities to antagonize further unfolding and subsequent degradation of these substrates (Martin et al. 2008; Nager et al. 2011). Sensitivity to ATP involves the specific unfolding pathway of a protein, rather than its global stability, and this pathway can vary for each substrate (Nager et al. 2011). Most substrates only require minimal ClpXP ATP hydrolysis rates for engagement, but some substrates require ClpXP to be fully bound with ATP for degradation to proceed. ATP hydrolysis rates can thus be used to differentially regulate protein levels through proteolysis (Martin et al. 2008).

What, then, is the function of the adaptor SprE? Since the degradation reaction requires SprE to deliver RpoS to ClpXP, the protein was included in our in vitro reactions. It may play some role in making degradation rates depend on ATP levels. However, SprE does not have any obvious ATP-binding motifs and is therefore unlikely to bind ATP directly. A more likely role for SprE may be to modulate RpoS levels during other stresses besides carbon starvation. For example, under DNA-damaging conditions, when ATP levels are high, RpoS is still stabilized due to the anti-adaptor protein IraD, which binds to SprE. It is thought that Ira-like proteins interfere with SprE, preventing ternary complex formation and subsequent degradation (Bougdour et al. 2008; Merrikh et al. 2009). Thus, SprE provides an input for additional levels of control.

The model that RpoS degradation is directly controlled by ATP levels suggests a general paradigm for protein stability in stationary phase. To date, most of the work on the ClpXP substrates has focused on sequence tags and adaptor proteins, but the remodeling of the proteome during stationary phase involves numerous ClpXP substrates, many of which do not have identified tags or adaptors. It is possible that our model links nutrient starvation to the unfolding properties of other proteins, which in turn may help to define the stationary phase proteome.

\section{Materials and methods}

\section{Bacterial strains, media, and growth conditions}

Standard microbiological techniques were used for strain construction (Supplemental Material; Silhavy et al. 1984). For exit from carbon starvation experiments, cultures were grown overnight in $0.4 \%$ glucose M63, diluted 1:100 in fresh medium (containing $0.005 \%$ pyruvate if the exit carbon source was pyruvate), and grown until the $\mathrm{OD}_{600}$ was $\sim 0.3-0.4$. Then cells were pelleted for $7 \mathrm{~min}$ in $15-\mathrm{mL}$ conical tubes at $1800 \mathrm{~g}$ in a Durafuge 200 centrifuge (Precision Scientific), washed twice in prewarmed medium lacking carbon, and incubated with aeration at $37^{\circ} \mathrm{C}$ (Ruiz and Silhavy 2003). After $60 \mathrm{~min}$ of starvation, the half-lives of the proteins during the exit from starvation were determined as described below. For purine depletion experiments, mutant strains with either purA::kan or guaB::kan were grown in minimal medium supplemented with $1 \mathrm{mM}$ adenine or guanine, respectively, until mid-log phase. Strains were washed as described above in full carbon medium lacking the purine and depleted for $1 \mathrm{~h}$, during which growth slowed down as measured by $\mathrm{OD}_{600}$, before measuring RpoS stability. The nicotinic acid depletion was carried out with the same method, except that the strains were grown in $10 \mu \mathrm{g} / \mathrm{mL}$ nicotinic acid and depleted for $2 \mathrm{~h}$ before measuring protein stability.

\section{Stability assays}

Chloramphenicol (final concentration $1.36 \mathrm{mg} / \mathrm{mL}$ ) was added $1 \mathrm{~min}$ before taking the $t=0$ time point. After that, the carbon source-either glucose or pyruvate (final concentration $0.4 \%$ ) - was added, and samples were taken at the times indicated (while the cultures were incubated at $37^{\circ} \mathrm{C}$ ) and processed for Western blot analysis (Supplemental Material).

\section{RpoS in vitro degradation assay}

Proteins were purified and in vitro RpoS degradation reactions were carried out according to previously described protocols with the modifications described below and in the Supplemental Material (Kim et al. 2000; Zhou et al. 2001). Endpoint samples were taken after the SprE/RpoS/ $\mathrm{ClpXP}$ mix was incubated for $16 \mathrm{~h}$ at $30^{\circ} \mathrm{C}$ with different concentrations of ATP (ranging from 0 to $400 \mu \mathrm{M}$ ) and a creatine kinase $(5 \mathrm{mM}) /$ creatine phosphate $(50 \mu \mathrm{g})$ ATP regeneration mix. For the kinetic experiments, the $\mathrm{SprE} / \mathrm{RpoS} / \mathrm{ClpXP}$ mix was incubated at $30^{\circ} \mathrm{C}$ with an ATP mix that included different combinations of ATP and ATP $\gamma \mathrm{S}$ in a final concentration of $5 \mathrm{mM}$ and no ATP regeneration mix (see the Supplemental Material for details).

\section{Acknowledgments}

We are very grateful to Tahmeena Chowdhury, Aliaa Abdelhakim, and Shamsah Ebrahim for all their guidance and help with the in vitro experiments. We also thank Drew Nager and Natividad Ruiz for helpful conversation. This work was supported by grants from the NIGMS to T.J.S. (GM065216) and T.A.B. (GM049224). T.A.B. and I.L. are employees of the Howard Hughes Medical Institute.

\section{References}

Baker TA, Sauer RT. 2012. ClpXP, an ATP-powered unfolding and proteindegradation machine. Biochim Biophys Acta 1823: 15-28.

Ballesteros M, Fredriksson A, Henriksson J, Nyström T. 2001. Bacterial senescence: Protein oxidation in non-proliferating cells is dictated by the accuracy of the ribosomes. EMBO I 20: 5280-5289.

Becker G, Klauck E, Hengge-Aronis R. 2000. The response regulator RssB, a recognition factor for $\sigma^{\mathrm{S}}$ proteolysis in Escherichia coli, can act like an anti- $\sigma^{\mathrm{s}}$ factor. Mol Microbiol 35: 657-666.

Bougdour A, Wickner S, Gottesman S. 2006. Modulating RssB activity: IraP, a novel regulator of $\sigma^{\mathrm{s}}$ stability in Escherichia coli. Genes Dev 20: 884-897.

Bougdour A, Cunning C, Baptiste PJ, Elliott T, Gottesman S. 2008. Multiple pathways for regulation of $\sigma^{\mathrm{S}}$ (RpoS) stability in Escherichia coli via the action of multiple anti-adaptors. Mol Microbiol 68: 298-313.

Burton RE, Baker TA, Sauer RT. 2003. Energy-dependent degradation: Linkage between ClpX-catalyzed nucleotide hydrolysis and proteinsubstrate processing. Protein Sci 12: 893-902.

Carabetta VJ, Mohanty BK, Kushner SR, Silhavy TJ. 2009. The response regulator SprE (RssB) modulates polyadenylation and mRNA stability in Escherichia coli. J Bacteriol 191: 6812-6821.

Cunning C, Elliott T. 1999. RpoS synthesis is growth rate regulated in Salmonella typhimurium, but its turnover is not dependent on acetyl phosphate synthesis or PTS function. J Bacteriol 181: 4853-4862.

Damerau K, St John AC. 1993. Role of Clp protease subunits in degradation of carbon starvation proteins in Escherichia coli. J Bacteriol 175: 53-63.

Deutscher J, Francke C, Postma PW. 2006. How phosphotransferase system-related protein phosphorylation regulates carbohydrate metabolism in bacteria. Microbiol Mol Biol Rev 70: 939-1031.

Dong T, Schellhorn HE. 2009. Global effect of RpoS on gene expression in pathogenic Escherichia coli O157:H7 strain EDL933. BMC Genomics 10: 349. doi: 10.1186/1471-2164-10-349.

Fischer D, Teich A, Neubauer P, Hengge-Aronis R. 1998. The general stress $\sigma$ factor $\sigma^{\mathrm{s}}$ of Escherichia coli is induced during diauxic shift from glucose to lactose. J Bacteriol 180: 6203-6206.

Fredriksson A, Ballesteros M, Peterson CN, Persson O, Silhavy TJ, Nyström T. 2007. Decline in ribosomal fidelity contributes to the accumulation and stabilization of the master stress response regulator $\sigma^{\mathrm{S}}$ upon carbon starvation. GenesDev 21: 862-874. 
Gigliobianco T, Lakaye B, Wins P, El Moualiij B, Zorzi W, Bettendorff L. 2010. Adenosine thiamine triphosphate accumulates in Escherichia coli cells in response to specific conditions of metabolic stress. BMC Microbiol 10: 148. doi: 10.1186/1471-2180-10-148.

Hersch GL, Burton RE, Bolton DN, Baker TA, Sauer RT. 2005. Asymmetric interactions of ATP with the $\mathrm{AAA}^{+} \mathrm{ClpX6}$ unfoldase: Allosteric control of a protein machine. Cell 121: 1017-1027.

Kim YI, Burton RE, Burton BM, Sauer RT, Baker TA. 2000. Dynamics of substrate denaturation and translocation by the ClpXP degradation machine. Mol Cell 5: 639-648.

Klauck E, Lingnau M, Hengge-Aronis R. 2001. Role of the response regulator RssB in $\sigma$ recognition and initiation of $\sigma$ proteolysis in Escherichia coli. Mol Microbiol 40: 1381-1390.

Kwon YK, Higgins MB, Rabinowitz JD. 2010. Antifolate-induced depletion of intracellular glycine and purines inhibits thymineless death in E. coli. ACS Chem Biol 5: 787-795.

Loewen PC, Hengge-Aronis R. 1994. The role of the $\sigma$ factor $\sigma^{\mathrm{S}}(\mathrm{KatF})$ in bacterial global regulation. Annu Rev Microbiol 48: 53-80.

Mandel MJ, Silhavy TJ. 2005. Starvation for different nutrients in Escherichia coli results in differential modulation of RpoS levels and stability. J Bacteriol 187: 434-442.

Martin A, Baker TA, Sauer RT. 2008. Protein unfolding by a $\mathrm{AAA}^{+}$ protease is dependent on ATP-hydrolysis rates and substrate energy landscapes. Nat Struct Mol Biol 15: 139-145.

McCann MP, Kidwell JP, Matin A. 1991. The putative $\sigma$ factor KatF has a central role in development of starvation-mediated general resistance in Escherichia coli. J Bacteriol 173: 4188-4194.

Merrikh H, Ferrazzoli AE, Bougdour A, Olivier-Mason A, Lovell ST. 2009. A DNA damage response in Escherichia coli involving the alternative $\sigma$ factor, RpoS. Proc Natl Acad Sci 106: 611-616.

Muffler A, Fischer D, Altuvia S, Storz G, Hengge-Aronis R. 1996. The response regulator RssB controls stability of the $\sigma^{\mathrm{S}}$ subunit of RNA polymerase in Escherichia coli. EMBO J 15: 1333-1339.

Murray HD, Schneider DA, Gourse RL. 2003. Control of rRNA expression by small molecules is dynamic and nonredundant. Mol Cell 12: 125-134.

Nager AR, Baker TA, Sauer RT. 2011. Stepwise unfolding of a $\beta$ barrel protein by the $\mathrm{AAA}^{+}$ClpXP protease. J Mol Biol 413: 4-16.

Peterson CN, Ruiz N, Silhavy TJ. 2004. RpoS proteolysis is regulated by a mechanism that does not require the $\operatorname{SprE}(\mathrm{RssB})$ response regulator phosphorylation site. J Bacteriol 186: 7403-7410.

Pratt LA, Silhavy TJ. 1996. The response regulator SprE controls the stability of RpoS. Proc Natl Acad Sci 93: 2488-2492.

Rice CD, Pollard JE, Lewis ZT, McCleary WR. 2009. Employment of a promoter-swapping technique shows that PhoU modulates the activity of the PstSCAB2 ABC transporter in Escherichia coli. Appl Environ Microbiol 75: 573-582.

Ruiz N, Silhavy TJ. 2003. Constitutive activation of the Escherichia coli Pho regulon upregulates rpoS translation in an Hfq-dependent fashion. J Bacteriol 185: 5984-5992.

Schneider DA, Gourse RL. 2004. Relationship between growth rate and ATP concentration in Escherichia coli: A bioassay for available cellular ATP. J Biol Chem 279: 8262-8268

Schweder T, Lee KH, Lomovskaya O, Matin A. 1996. Regulation of Escherichia coli starvation $\sigma$ factor $\left(\sigma^{\mathrm{s}}\right)$ by ClpXP protease. J Bacteriol 178: $470-476$.

Silhavy TJ, Berman ML, Enquist LW. 1984. Experiments with gene fusions. Cold Spring Harbor Laboratory, Cold Spring Harbor, NY.

Weber H, Polen T, Heyveling J, Wendisch VF, Hengge R. 2005. Genomewide analysis of the general stress response network in Escherichia coli: $\sigma^{\mathrm{s}}$-dependent genes, promoters, and $\sigma$ factor selectivity. J Bacteriol 187: 1591-1603.

Zhang S, Haldenwang WG. 2005. Contributions of ATP, GTP, and redox state to nutritional stress activation of the Bacillus subtilis $\sigma^{\mathrm{B}}$ transcription factor. J Bacteriol 187: 7554-7560.

Zhou Y, Gottesman S. 2006. Modes of regulation of RpoS by H-NS. I Bacteriol 188: 7022-7025.

Zhou Y, Gottesman S, Hoskins JR, Maurizi MR, Wickner S. 2001. The RssB response regulator directly targets $\sigma^{\mathrm{S}}$ for degradation by ClpXP. Genes Dev 15: 627-637. 


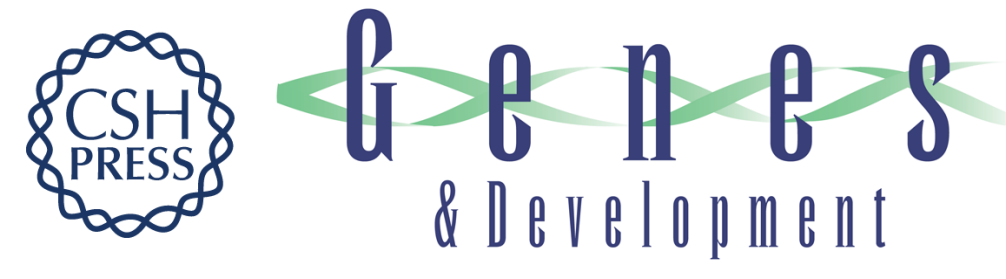

\section{RpoS proteolysis is controlled directly by ATP levels in Escherichia coli}

Celeste N. Peterson, Igor Levchenko, Joshua D. Rabinowitz, et al.

Genes Dev. 2012, 26:

Access the most recent version at doi:10.1101/gad.183517.111

\section{Supplemental http://genesdev.cshlp.org/content/suppl/2012/03/16/26.6.548.DC1 Material}

References This article cites 36 articles, 21 of which can be accessed free at: http://genesdev.cshlp.org/content/26/6/548.full.html\#ref-list-1

\section{License}

Email Alerting

Receive free email alerts when new articles cite this article - sign up in the box at the top Service 